\title{
Analysis of LCoS displays performance in diffractive optics
}

\author{
A. Lizana ${ }^{1}$, L. Lobato $^{1}$, C. Iemmi ${ }^{2}$, A. Márquez ${ }^{3}$, I. Moreno $^{4}$, J. Campos $^{1}$ and M.J. Yzuel ${ }^{1}$ \\ ${ }^{1}$ Dept. of Physics, Universitat Autònoma de Barcelona, Bellaterra, Spain \\ ${ }^{2}$ Dept. of Physics, Universidad de Buenos Aires, Buenos Aires, Argentina \\ ${ }^{3}$ Dept. of Physics, Ing. Sistemas y Teoría de la Señal, Univ. Alicante, Alicante, Spain \\ ${ }^{4}$ Dept. de Ciencia de Materiales, Óptica y Tecnología de la Señal, Univ. Miguel Hernandez, Elche, Spain
}

\begin{abstract}
In this paper, we describe the Mueller-Jones combined method which is useful to optimize the LCoS displays phase response. This method, by means of the experimentally obtained Mueller matrices of the device, enables to obtain pairs of states of polarization (for the generation and for the detection states), which lead to the phase-only modulation regime. Moreover, some experimental results are provided as a function of the incident angle, wavelength and gray level. In addition, we also show the strong dependence of the LCoS performance with the signal addressed to the device, which affects the value of different physical parameters, such as the global phase-shift or the time-fluctuations in phase. Retardance curve and time-fluctuations in phase for the different sequences studied are obtained from the experimental Mueller matrices (the former) and by using a diffractive based set-up (the latter). The efficiency of basic diffractive optical elements is tested with the LCoS display, emphasizing the suitability of the best electrical sequence found when used in diffractive optics.
\end{abstract}

\section{Introduction}

Liquid Crystal on Silicon (LCoS) devices are a type of Liquid Crystal Display (LCD) working by reflection. LCoS displays show a great spatial light modulation capability that make them very useful when spatially manipulating optical properties of light beams. As a consequence, LCoS displays are nowadays widespread used in many optical applications as for instance, in diffractive optics [1], polarimetry [2] or medical physics [3].

As it is well-known, the efficiency of only-phase diffractive optical elements (DOEs) addressed to the LCoS display is improved if the device is optimized in the only-phase regime. As a consequence, a mathematical model providing maximum phase-shift and constant transmission becomes necessary for its optimization. However, LCoS displays performance also depends on the electrical pulse scheme (electrical sequence) addressed to it $[4,5]$. Therefore, when a set of possible electrical schemes is available, an optimization for every scheme used is required.

In this paper, in order to provide higher insight on this topic, we describe a combined method useful to optimize the LCoS display response in the phase-only regime. Moreover, some experimental results as a function of the incident angle, the wavelength and the gray level, are provided. In addition, we also conduct a thoroughly study of LCoS displays performance as a function of different electrical sequences, showing a great influence in some physical parameters as the global phase-shift or the phase-fluctuations in time.

The outline of this work is as it follows. In section 2, a procedure useful to optimize the phase response of LCoS diplays (the Mueller-Jones combined method) is briefly explained. In addition, some experimental results as a function of different physical parameters are also provided. In section 3, we study the phase response of a Parallel Aligned LCoS display as a function of different electrical schemes addressed to the device. Retardance curve and time-fluctuations in phase for the different sequences are experimentally obtained. Furthermore, the influence of the electrical signal scheme in the efficiency of binary gratings generated with the device is discussed. Finally, the conclusions are presented in section 4.

\section{LCoS display phase Optimization}

\subsection{Mueller-Jones combined method}

In this section, a combined method useful to optimize the LCoS display phase response is briefly described. More details can be found in Ref. [6]. This method is based on the combination of the Mueller-Stokes formalism [7] and the Jones formalism [8]. In this way, whereas the Mueller

This is an Open Access article distributed under the terms of the Creative Commons Attribution-Noncommercial License 3.0, which permits unrestricted use, distribution, and reproduction in any noncommercial medium, provided the original work is properly cited. 
formalism takes into account the LCoS effective depolarization contribution and enables to obtain polarimetric information just by performing radiometric measurements, the Jones formalism allows us to control the phase modulation introduced by the device.

Let us denote $M$ as the Mueller matrix of a given polarizing sample. By using the Lu-Chiman polar decomposition [7], the matrix $M$ can be written as the product of three elemental Mueller matrices: the depolarizer $M_{\Delta}$, the retarder $M_{R}$ and the diattenuator $M_{D}$.

$$
M=M_{\Delta} \cdot M_{R} \cdot M_{D}
$$

As LCoS displays have proved to be nondiattenuating devices [9], the matrix $M_{D}$ can be assumed as the identity and the Eq. (1) is therefore, reduced to the following expression:

$$
M=M_{R} \cdot M_{D}
$$

The Mueller matrix of the retarder $M_{R}$ is a unitary matrix describing part of the total information enclosed into the matrix $M$ of the LCoS display. As $M_{R}$ keeps some information of the fully polarized and non-absorbing contribution, it can be associated to the Jones matrix of an equivalent retarder $m_{R}$ by means of a Jones-Mueller mathematical transformation [7] (note that we use the minuscule $m$ to denote Jones matrices and difference them from Mueller matrices $M$ ). In this way, we are able to obtain the $A$ and $B$ complex parameters of the following $2 \times 2$ unimodular unitary Jones matrix, describing non-absorbing reciprocal polarization devices (for which the LCoS is a particular case) [10]:

$$
m_{R}=e^{-i \beta}\left(\begin{array}{cc}
A & B \\
-B^{*} & A^{*}
\end{array}\right)
$$

where the $\operatorname{sign} *$ denotes the complex conjugate. Note that the external phase $\beta$ shown in Eq. (3), which in general depends on the gray level, can not be obtained from the Jones-Mueller transformation and consequently, an experimental calibration is required.

The Mueller matrix of the LCoS display can be readily obtained by using a polarimeter based set-up [9]. Thus, by using an experimental Muller matrix and the polar decomposition method, the corresponding parameters $A$ and $B$ of the equivalent retarder are calibrated.

The fully polarized part of the state of polarization (SOP) of a light beam exiting from the LCoS display can be described by a Jones vector, let us call it $J_{\text {lcos }}$. The $J_{\text {lcos }}$ vector can be obtained by multiplying the Jones vector of the incident beam $J_{\mathrm{i}}$ by the matrix $m_{R}$ shown in Eq. (3). Afterward, if projecting the $J_{\text {lcos }}$ vector upon a detection configuration (SOP defined by a polarization state detector (PSD) configuration and described by the Jones vector $J_{P S D}$ ), we obtain a complex number $z$ whose modulus corresponds to the intensity behind the PSD configuration and whose argument relates to the phase.

$$
z=J_{P S D} \cdot J_{l \cos }=J_{P S D} \cdot m_{R} \cdot J_{i}
$$

Then, the global phase of the exiting beam $\delta$ (i.e. the phase of the complex number $z$ ) is the summation of two contributions:

$$
\boldsymbol{\delta}=\boldsymbol{\beta}+\boldsymbol{\phi}
$$

where $\phi$ depends on the $A$ and $B$ parameters and on the azimuth and ellipticity angles [7] determining $J_{i}$ and $J_{P S D}$ Jones vectors. As previously stated, the external phase $\beta$ is unknown. Therefore, by performing experimental measurements of the global phase $\delta$ (for instance, by using an interferometric set-up [6]) for known values of the generated and analyzer states of polarization (selected respectively by using a polarization state generator (PSG) and a polarization state detector (PSD)), the phase $\beta$, which is fixed for a given gray level, is obtained. Once the phase $\beta$ and the complex parameters $A$ and $B$ are calibrated, by using Eq. (4) and for a given generated and detected SOPs, the phase of the beam exiting from the PSD system is obtained.

Finally, the optimization of the LCoS display phase response is achieved by using a software that allows to search a pair of generated and analyzer SOPs which maximize a given figure of merit $Q$. In particular, we have used the following figure of merit which enables to obtain the maximum transmission and global phase-shift while a minimum intensity contrast is simultaneously obtained, being this profile very attractive in diffractive optics.

$$
Q=\frac{1}{\lambda_{1}+\lambda_{2}+\lambda_{3}}\left[\lambda_{1} \frac{\Delta \tau}{2 \pi}+\lambda_{2}(1-\Delta T)+\lambda_{3} T_{m}\right]
$$

where $\Delta \tau$ is the global phase-shift, $\Delta T$ is the difference between the maximum and minimum transmittance values, $T_{m}$ the minimum value of the transmittance and the coefficients $\lambda_{1}, \lambda_{2}, \lambda_{3}$ are the weights of these three parameters.

\subsection{Experimental results}

In this section, we show some phase-only optimization results obtained by using the Mueller-Jones combined method described in the section 2.1. For the LCoS display tested, the Mueller matrices have been obtained as a function of different incident angles, wavelengths and gray levels, by using the set-up and experimental procedure described in Ref. [9]. In particular, the LCoS display used is a Philips LCoS model X97c3A0, sold as the kit LC-R2500 by Holoeye. The LC-R2500 is a 2.46 $\mathrm{cm}$ diagonal monochrome reflective $\mathrm{LCoS}$ of the $45^{\circ}$ twisted nematic type, with XGA resolution (1024 x 768 pixels), with digital data input and digitally controlled gray scales with 256 gray levels. The pixels are square with a pixel center to center separation of $19 \mu \mathrm{m}$ and a fill factor of $93 \%$.

Figure 1 shows the phase (in brown and right axis) and intensity (in pink and left axis) values as a function of the gray level when performing an optimization in quasi-normal incidence (4 degree between the incident and the reflected beams) and by using a $633 \mathrm{~nm}$ wavelength. The lines show the theoretical values and the 
spots are representing the experimental ones. In addition, in the optimization performance we have allowed to decrease the minimum intensity value in Eq. (6), leading to higher phase-shift.

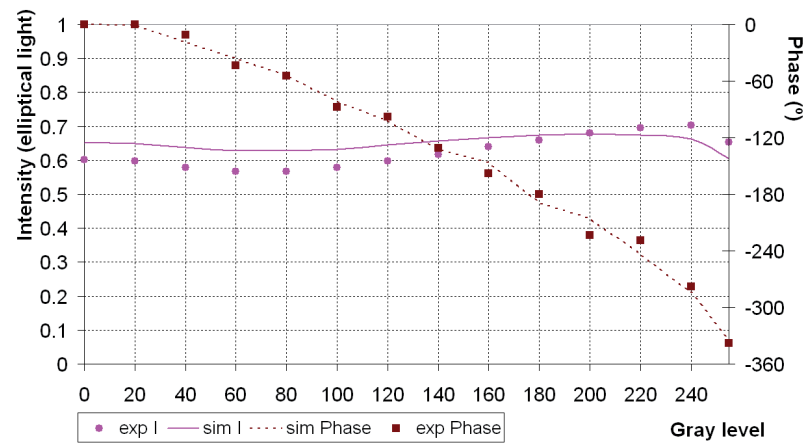

Fig. 1. Intensity and phase as a function of the gray level. The rotation angle values of polarizers and quarter-waveplates used at the PSG and PSD systems are: $\mathrm{P} 1=88^{\circ}$ and $\mathrm{WP} 1=7^{\circ} ; \mathrm{P} 2=90^{\circ}$ and $\mathrm{WP} 2=-15^{\circ}$

In Fig. 1, we see a global phase-shift close to $2 \pi$ at the same time that a flat intensity modulation is achieved, both of them represented as a function of the gray level. Moreover, we want to emphasize that the degree of polarization (DOP) [7] associated to this configuration is almost constant to 1 throughout all the gray level range (i.e. no effective depolarization is added to the system). This is a very attractive configuration for diffractive optics, obtaining a large phase modulation even when using a large wavelength. In addition, the great agreement between theory and experimental results, clearly highlights the suitability of the optimization method applied.

Next, we have studied how the light beam wavelength influences on the LCoS display performance. First, we have set the same SOPs configuration obtained with the previous optimization and we have used shorter wavelengths $(532 \mathrm{~nm}$ and $458 \mathrm{~nm}$ ). The results show a coupled intensity and phase as a function of the gray level, resulting in a degraded LCoS display phase-only behaviour [11].

After this, a specific LCoS display optimization for every wavelength used should be implemented. In this sense, Fig. 2 and 3 show the results obtained when optimizing the LCoS display for the $532 \mathrm{~nm}$ and $458 \mathrm{~nm}$ wavelengths, and in both cases, under quasi-normal incidence.

We can easily notice that, the shorter the wavelength, the larger the phase-shift ( $2 \pi$ for $532 \mathrm{~nm}$ and 480 degrees for $458 \mathrm{~nm}$ ). Nevertheless, some measurements have shown that the degree of polarization can be decreased when using shorter wavelengths, which may also decrease the efficiency of the applications where the LCoS display is being used, and so, DOP must be also taken into account.

Finally, we have studied the influence of the incident angle on the LCoS display performance. To do this, we have fixed a light source of $633 \mathrm{~nm}$ and we have measured the Mueller matrix of the LCoS display for different angles of incidence.

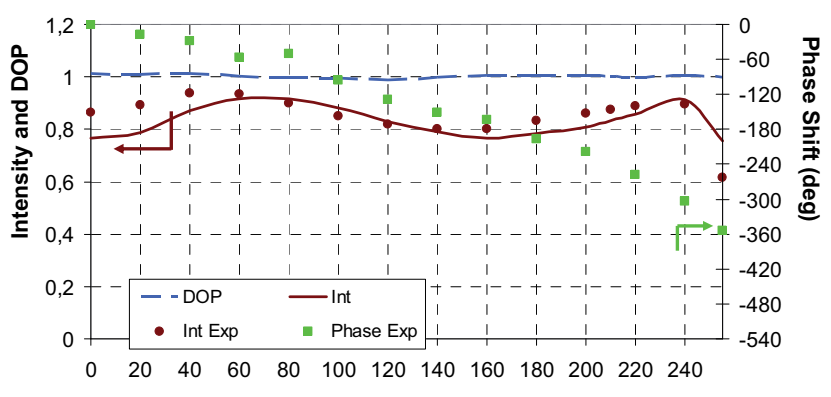

Fig. 2. Intensity (in brown and left axis), DOP (in blue and left axis) and phase (in green and right axis) as a function of the gray level. The rotation angle values of polarizers and quarterwaveplates used at the PSG and PSD systems are: $\mathrm{P} 1=82^{\circ}$ and $\mathrm{WP} 1=72^{\circ} ; \mathrm{P} 2=121^{\circ}$ and $\mathrm{WP} 2=126^{\circ}$

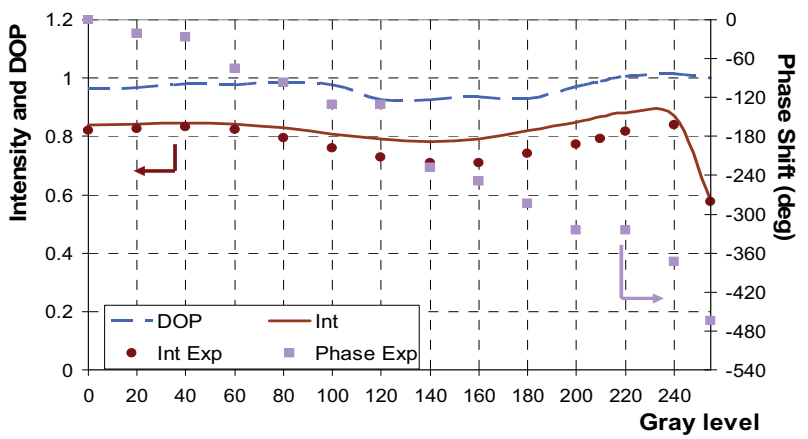

Fig. 3. Intensity (in brown and left axis), DOP (in blue and left axis) and phase (in violet and right axis) as a function of the gray level. The rotation angle values of polarizers and quarterwaveplates used at the PSG and PSD systems are: $\mathrm{P} 1=78^{\circ}$ and $\mathrm{WP} 1=73^{\circ} ; \mathrm{P} 2=257^{\circ}$ and $\mathrm{WP} 2=263^{\circ}$

Figure 4 shows the global phase-shift and the intensity values as a function of the gray level when optimizing the system for an incident angle equal to 12.5 degrees. We can notice that the global phase-shift is slightly lower than the one shown in Fig. 1, obtained for quasi-normal incidence. However, both results can be considered quite similar. In addition, in Ref. [13] is proved as for small incident angle variations (about 10 degrees) a unique optimization is enough. Unlikely, results shown in Fig. 5 (where an incident angle of 45 degrees is undertaken) are quite different, obtaining a notably reduction in the LCoS display phase-shift response (about 240 degree).

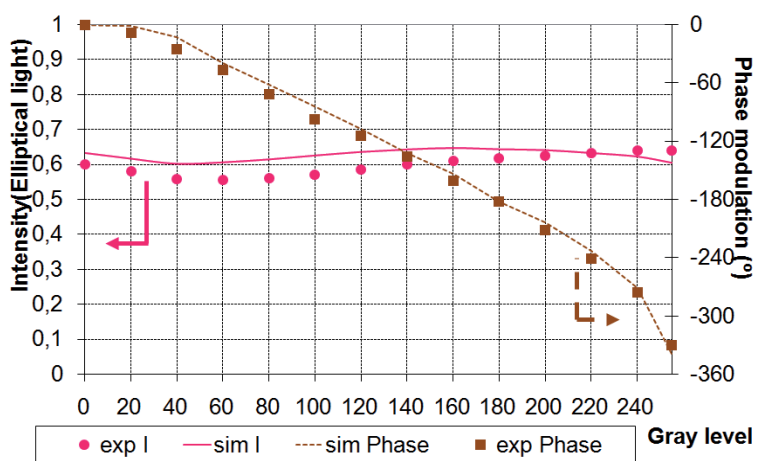

Fig. 4. Intensity (in pink and left axis) and phase (in brown and right axis) as a function of the gray level. The rotation angle values of polarizers and quarter-waveplates used at the PSG and PSD systems are: $\mathrm{P} 1=95^{\circ}$ and $\mathrm{WP} 1=85^{\circ} ; \mathrm{P} 2=-299^{\circ}$ and $\mathrm{WP} 2=-296^{\circ}$ 


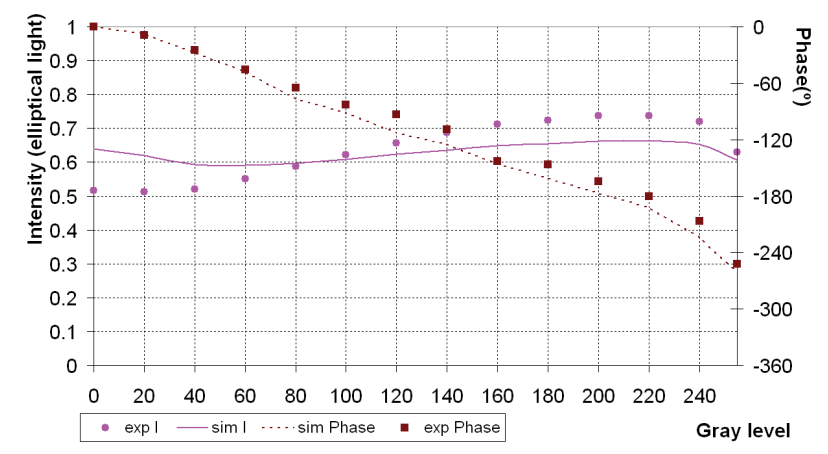

Fig. 5. Intensity (in pink and left axis) and phase (in brown and right axis) as a function of the gray level. The rotation angle values of polarizers and quarter-waveplates used at the PSG and PSD systems are: $\mathrm{P} 1=91^{\circ}$ and $\mathrm{WP} 1=104^{\circ} ; \mathrm{P} 2=54^{\circ}$ and $\mathrm{WP} 2=53^{\circ}$

However, there are some applications involving high numerical apertures, where a wide range of incident angles are used, that also require large phase-shift (as for instance in optical trappings set-ups [12]). In such as applications, the use of a characterizing set-up where a beam-splitter is included (as the set-up proposed in Ref. [13]) it is recommended, leading to better efficiency than set-ups with large angles between the incident and reflected beams.

\section{Influence of the electrical signal in the LCoS display performance}

In this section, we analyze how the LCoS performance is influenced by the electrical signal scheme addressed to the device. In general, different electrical signals provide different polarimetric responses of the LCoS display, as for instance, the global phase-shift obtained. However, it has been shown that in digital addressing LCoS displays, electrical configurations providing large phase-shift (which is very interesting in order to obtain high efficiency for the generated diffractive elements) may introduce fluctuations of the liquid crystal (LC) molecules as a function of the time. As a consequence, these time-fluctuations of the LC molecules result in two proved physical effects that may reduce the efficiency in some applications. In particular, they are the origin of the effective depolarization added by some LCoS devices $[9,14,15]$ and of the phase fluctuations phenomena [16, 17].

A type of LCoS display is the Parallel Aligned (PA) LCoS display. In these devices, the major axes of their LC molecules are parallel oriented. As a consequence, by using linear polarized light in the direction of the LC extraordinary axis, it is possible to work with the onlyphase regime without any effective depolarization added to the LCoS display reflected beam. However, in presence of time-fluctuations of the LC molecules, phase fluctuations in time are always present.

In order to analyze the influence of the electrical signal in the phase response of a LCoS dispay, we have used a PA LCoS display that allows to address different binary pulsed schemes (electrical sequences). More technical details about the electrical sequences used can be found in Ref. [4]. In addition, the PA LCoS display used is an electrically controlled birefringence (ECB) LCoS display distributed by the company HOLOEYE. It is an active matrix reflective mode device with 1920x1080 pixels and 0.7" diagonal named the PLUTO Spatial Light Modulator (SLM). The pixel pitch is of 8.0 $\mu \mathrm{m}$ and the display has a fill factor of $87 \%$. The signal is addressed via a standard DVI (Digital Visual Interface) signal. We have used the PA LCoS display operating in only-phase mode by properly using incident linear polarized light. First, we have measured the global phase (i.e. the averaged phase [16]) provided by the device, as a function of the gray level and for three different sequences, labelled in this work as "18-6 default", "55(1064)" and "5-5(543)". The phase values have been obtained by using the experimentally measured Mueller matrices of the device and by applying the Jones-Mueller combined method explained in section 2 .

Figure 6 shows the retardance values as a function of the gray level for the three different sequences. Whereas sequences "18-6 default" (in blue) and "5-5(1064)" (in red) show large phase modulation as a function of the gray level (obtaining respectively a global phase-shift of 520 and 480 degrees) the sequence "5-5(543)" (in green) provide a notably shorter phase modulation (about 280 degrees). This result is logical when taking into account that sequences "18-6 default" and "5-5(1064)" are optimized for operating in the infrared range and the " 5 $5(543)$ " sequence is optimized for the $543 \mathrm{~nm}$ wavelength. This means that a sequence designed for a specific wavelength gives a phase depth of approximately $2 \pi$ radians for this wavelength.

Note that the wavelength we use, $633 \mathrm{~nm}$, does not correspond to any of the ones for which the sequences we use have been designed. We have chosen this wavelength because we are interested in analyzing the effect on the LCoS display performance when considering different configurations between phase fluctuations and global phase-shift. This can be achieved by using different electrical sequences for a unique wavelength.

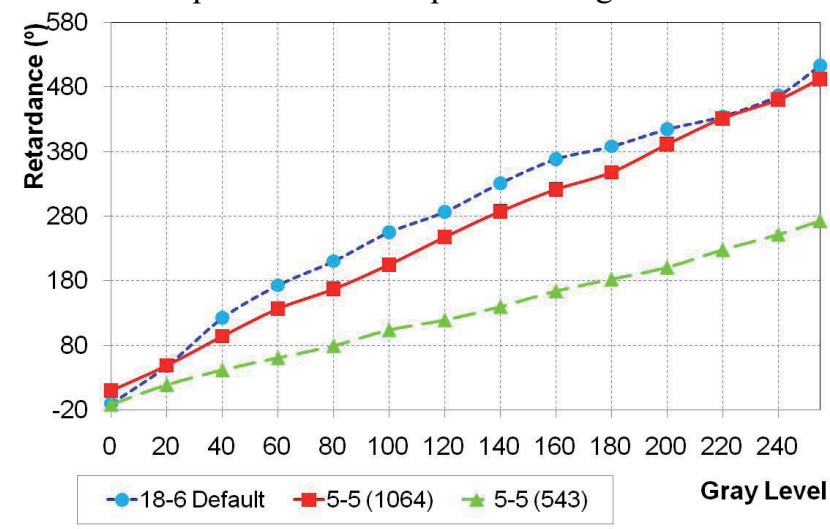

Fig. 6. Retardance curves for the three electrical schemes used.

Therefore, we have measured the phase response of the device as a function of the time for the three different sequences previously stated. For this aim, we have used the diffractive set-up reported in Ref.[16]. The obtained results are plotted in Fig. 7. 


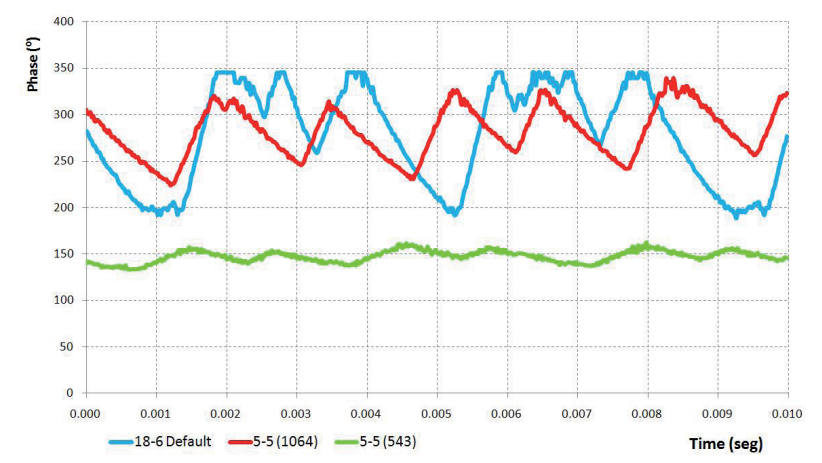

Fig. 7. Intensity and phase as a function of the gray level.

Figure 7 shows large amplitude of phase fluctuations for the"18-6 default" and "5-5(1064)" sequences, being larger for the first one. Unlikely, "5-5(543)" sequence provide an almost flat phase evolution as a function of the time. However, this last sequence is providing the shorter phase-shift (see Fig. 6).

Finally, we have tested the influence of these three electrical signals in the efficiency of generated basic diffractive optical elements (DOEs). In fact, we have addressed binary gratings with an averaged phase-shift equal to 200 degree. The results for the three sequences used are respectively plotted in Fig. (8-10), where the normalized intensity of the diffracted orders zero (in blue) and one (in pink) are represented as a function of the time.

On the one hand, in Fig. 8 and 9, we observe that "18-6 default" and "5-5(1064)" sequences result in a bad efficiency of the displayed DOEs, due to their high timefluctuations of the phase (Fig. 7). We want to empathize that the efficiency degradation occurs even when considering two sequences providing a large phase-shift (Fig. 6). On the other hand, sequence "5-5(543)" gives a much better efficiency of the displayed DOEs, even if the phase-shift is notably shorter than $2 \pi$. Therefore, the effect of large phase-fluctuations strongly affects the efficiency of DOEs, being possible to obtain better results with an electrical sequence providing shorter phase-shift but with a controlled phase-fluctuations phenomena.

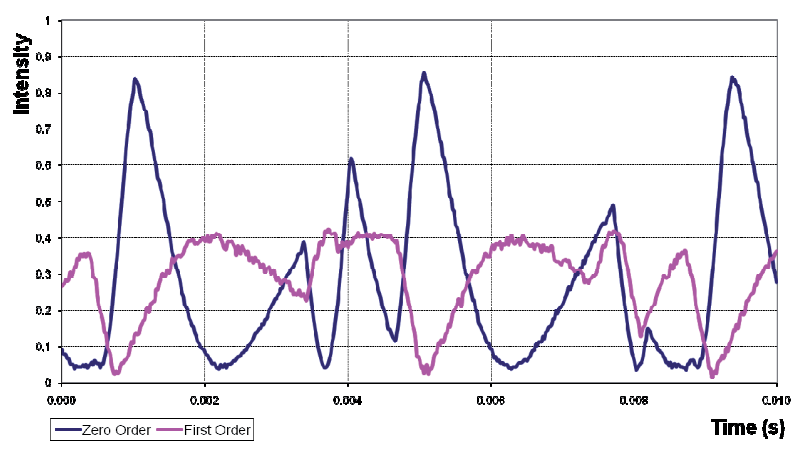

Fig. 8. Intensity as a function of the time of the zero (in blue) and the first (in pink) diffracted orders for a binary grating with a phase-shift to 200 degrees and by addressing the " $18-6$ default" sequence.

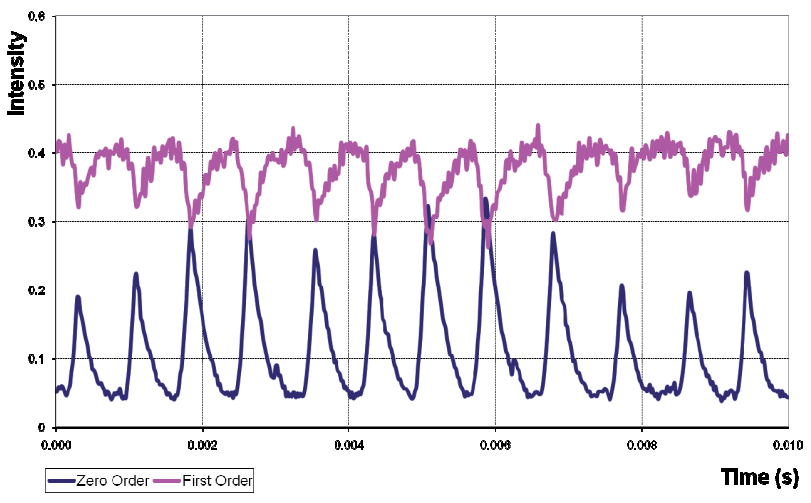

Fig. 9. Intensity as a function of the time of the zero (in blue) and the first (in pink) diffracted orders for a binary grating with a phase-shift to 200 degrees and by addressing the " $5-5(1064)$ " sequence.

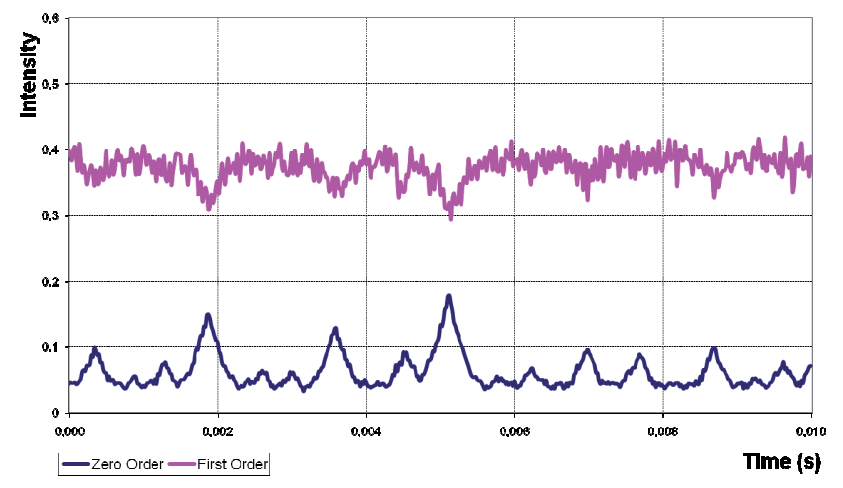

Fig. 10. Intensity as a function of the time of the zero (in blue) and the first (in pink) diffracted orders for a binary grating with a phase-shift to 200 degrees and by addressing the "5-5(543)" sequence.

In this framework, equilibrium between large phase-shift and reduced phase fluctuations in time becomes important in order to maximize the efficiency in diffractive optics. Thus, a mathematical model suitable to quantitatively evaluate the efficiency of DOEs as a function of these to parameters becomes very useful in diffractive optic applications [5].

\section{Conclusions}

This paper provides with an in-depth study of important factors determining the LCoS efficiency in diffractive optics.

Firstly, we have presented the Mueller-Jones method suitable for phase response optimization. Agreement between theory and experimental results, totally prove the effectiveness of the aforesaid method.

Other parameters influencing the LCoS performance efficiency comprise the wavelength and the angle of incidence of the light beam. Shorter wavelengths often result in a larger phase-shift response although they may also produce higher depolarization. Regarding the angle of incidence, small incidence angles result in larger phase-shift LCoS display response. 
Finally, we have studied the modulator efficiency dependence on the electrical sequence addressed to the device. Experimental results show that the efficiency of DOEs can be enhanced when addressing a sequence with a shorter phase-shift but controllable phase-fluctuations phenomena.

\section{References}

1. J. Turunen and F. Wyrowski, Diffractive Optics for Industrial and Commercial Applications, Akademie Verlag, Berlin, (1997)

2. A. De Martino, Y.K. Kim, E. Garcia-Caurel, B.Laude and B.Drévillon, Opt. Letters, 28 (2003).

3. M. Anastasiadou, A.De Martino, D. Clement, F.Liège, B.Laude-Boulesteix, N.Quang, J. Dreyfuss, B.Huynh, A. Nazac, L.Schwartz and H.A.S. Cohen, Phy. Stat. Sol., 5 (2008)

4. A. Hermerschmidt, S. Osten, S. Krüger and Thomas Blümel, Proc. SPIE, 6584 (2007).

5. A. Lizana, A. Márquez, L. Lobato, Y. Rodange, I. Moreno,C. Iemmi and J. Campos, sent to Opt. Express.

6. I. Moreno, A. Lizana, J. Campos, A. Márquez, C. Iemmi and M. J. Yzuel, Opt. Letters. 33 (2008).

7. D. Goldstein, Polarized Light, Marcel Dekker, NY, (2003).

8. R. C. Jones, J. Opt. Soc. Am. A 31(1941).

9. A. Márquez, I. Moreno, C. Iemmi, A. Lizana, J. Campos and M. J. Yzuel, Opt. Express 16, (2008).

10. I. Moreno, P. Velásquez, C. R. Fernández-Pousa, M. M. Sánchez-López and F. Mateos, J. Appl. Phys. 94, (2003).

11. A. Lizana, A. Márquez, I. Moreno, C. Iemmi, J. Campos and M. J. Yzuel, J. Eur. Opt. Soc. - Rapid Pub. 3 (2008).

12. E. Martín-Badosa, M. Montes-Usategui, A. Carnicer, J. Andilla, E. Pleguezuelos, and I. Juvells, J. Opt. A Pure Appl. Op. 9 (2007).

13. A. Lizana, N. Martin, M. Estapé, E. Fernández, I. Moreno, A. Márquez, C. Iemmi, J. Campos and M. J. Yzuel, Opt. Express 17, (2009).

14. J.E. Wolfe and R. A. Chipman, Appl. Opt. 45 (2006).

15. A. Lizana, I. Moreno, C. Iemmi, A. Márquez, J. Campos and M.J.Yzuel, Appl. Opt. 47 (2008).

16. A. Lizana, I. Moreno, A. Márquez, C. Iemmi, E. Fernández, J. Campos and M.J.Yzuel, Opt. Express 16 (2008).

17. J. R. Moore, N. Collings, W. A. Crossland, A. B. Davey, M. Evans, A. M. Jeziorska, M. Komarčević, R. J. Parker, T. D. Wilkinson and H. Xu, IEEE Photon. Techol. Lett. 20 (2008).

\section{Acknowledgments}

We acknowledge financial support from the Spanish Ministerio de Educación y Ciencia (grants FIS200913955-C02-01 and FIS2009-13955-C02-02) and from Generalitat de Catalunya (2009PIV 00051). C. Iemmi gratefully acknowledges the support of the Universidad de Buenos Aires and CONICET (Argentina). 